\title{
Tecnoestrés en estudiantes de una universidad pública de la Amazonía peruana durante la pandemia COVID-19
}

\author{
DiD Edwin Gustavo Estrada Araoz ${ }^{1}$, (D) Néstor Antonio Gallegos Ramos ${ }^{2}$, (D Karl Herbert Huaypar Loayza ${ }^{3}$, (D) Yolanda \\ Paredes Valverde ${ }^{4}$, iD Rosel Quispe Herrera ${ }^{5}$ \\ 1 Universidad Nacional Amazónica de Madre de Dios. Departamento de Educación. Avenida Jorge Chávez $\mathrm{n}^{\circ} 1160$, Puerto \\ Maldonado. Madre de Dios, Perú. 2, 3, 4, 5 Universidad Nacional Amazónica de Madre de Dios, Perú.
}

Autor para correspondência: edwin5721@outlook.com

RESUMEN. La presente investigación tuvo como objetivo describir el tecnoestrés de los estudiantes de la carrera profesional de Educación de una universidad pública de la Amazonía peruana durante la pandemia COVID-19. El enfoque fue cuantitativo, el diseño no experimental y el tipo de investigación, descriptivo transversal. La muestra fue constituida por 232 estudiantes a quienes se les aplicó el Cuestionario de Tecnoestrés, instrumento con adecuados niveles de confiabilidad y validez. Los resultados indican que los estudiantes se caracterizaban por tener niveles moderados de tecnoestrés. Asimismo, respecto a sus factores, se encontró que existían niveles bajos de tecnoansiedad y niveles moderados de tecnoadicción y tecnofatiga. Por otro lado, se determinó que algunas variables sociodemográficas como el sexo, el grupo etario y la condición laboral de los estudiantes se asociaba significativamente a los niveles de tecnoestrés. Se concluyó que es necesario que la universidad tome algunas medidas preventivas y correctivas que permitan disminuir la prevalencia de dicho padecimiento en los estudiantes. Asimismo, sería importante fomentar la desconexión digital para que ellos realicen actividades físicas, de atención a sus familias y socialización.

Palabras clave: tecnoestrés, educación virtual, educación superior universitaria, estudiantes universitarios, COVID-19. 


\title{
Technostress in students of a public university in the Peruvian Amazon during the COVID-19 pandemic
}

\begin{abstract}
The present research aimed to describe the technostress of the students of the Education career of a public university in the Peruvian Amazon during the COVID-19 pandemic. The approach was quantitative, the design nonexperimental and the type of research, descriptive, crosssectional. The sample consisted of 232 students to whom the Technostress Questionnaire was applied, an instrument with adequate levels of reliability and validity. The results indicate that the students were characterized by having moderate levels of technostress. Likewise, regarding its factors, it was found that there were low levels of technoanxiety and moderate levels of technoaddiction and technofatigue. On the other hand, it was determined that some sociodemographic variables such as sex, age group and the work condition of the students were significantly associated with levels of techno-stress. It was concluded that it is necessary for the university to take some preventive and corrective measures to reduce the prevalence of this condition in the students. Likewise, it would be important to promote digital disconnection so that they carry out physical activities, care for their families and socialization.
\end{abstract}

Keywords: techno-stress, virtual education, university higher education, university students, COVID-19. 


\section{Tecnoestresse em estudantes de uma universidade pública da Amazônia peruana durante a pandemia COVID-19}

RESUMO. A presente pesquisa teve como objetivo descrever o tecnoestresse dos estudantes da carreira profissional de Educação de uma universidade pública da Amazônia Peruana durante a pandemia COVID-19. O enfoque foi quantitativo, o desenho não experimental e o tipo de pesquisa descritiva transversal. A amostra foi composta por 232 estudantes aos quais foi aplicado o Questionário de Tecnostresse, instrumento com níveis adequados de confiabilidade e validade. Os resultados indicam que os estudantes se caracterizaram por apresentar níveis moderados de tecnoestresse. Da mesma forma, quanto aos seus fatores, verificou-se que houve baixos níveis de tecnoansiedade e moderados níveis de tecnoadição e tecnofadiga. Por outro lado, constatou-se que algumas variáveis sociodemográficas como sexo, faixa etária e condição de trabalho dos estudantes estiveram significativamente associadas aos níveis de tecnoestresse. Concluiu-se que é necessário que a universidade tome algumas medidas preventivas e corretivas para reduzir a prevalência desta patologia nos estudantes. Da mesma forma, seria importante promover o desligamento digital para que possam realizar atividades físicas, cuidar da família e socializar.

Palavras-chave: tecnoestresse, educação virtual, ensino superior universitário, estudantes universitários, COVID-19. 


\section{Introducción}

En el mes de diciembre del año 2019 fueron reportados en la ciudad de Wuhan (China) casos de neumonía atípica ocasionados por un nuevo coronavirus al que le denominaron SARS-CoV-2 que a su vez causaba la enfermedad de COVID-19 (Mojica y Gonzales, 2020; Quiroz et al., 2020). Debido al incremento exponencial de casos en todo el mundo, dicha enfermedad fue catalogada como pandemia internacional en marzo del año 2020, contexto que puso a prueba a los sistemas sanitarios de todos los países (Buitrago et al., 2020). La pandemia, desde luego, generó un gran impacto en la sociedad debido a las repercusiones sociales, sanitarias, económicas, educativas, culturales, etc. (Lizcano y Arroyave, 2020; Murillo y Duk, 2020).

En el Perú, la pandemia de COVID19 ocasionó que el Gobierno declare el 15 de marzo del 2020 el estado de emergencia nacional y aislamiento social obligatorio como una medida excepcional para evitar que el virus se propague. Ello provocó que las actividades académicas presenciales en las instituciones educativas básicas y superiores fueran interrumpidas (Estrada et al., 2020a; Mejía et al., 2021) y que la modalidad cómo se venía brindando el servicio educativo migre, pasando de ser presencial a ser exclusivamente virtual
(Estrada y Mamani, 2021). Sin embargo, esta reforma educativa obligatoria generó mucha preocupación y presión puesto que muchos docentes como estudiantes debían afrontar una metodología poco practicada y para lo cual no se encontraban preparados (Suárez et al., 2021). Pese a ello, continuaron adelante, ya que existía la necesidad de que la prestación del servicio educativo no se vea interrumpida (Toquero, 2020; Ordorika, 2020).

La educación virtual demanda procesos tecnológicos y pedagógicos asequibles, sostenibles, dinámicos y versátiles para que pueda ser efectiva (Díaz et al., 2021). Cuando se da de manera planificada, puede facilitar e incluir a todas las personas que deseen aprender tomando en cuenta la flexibilidad de los horarios para concretizar diversos objetivos académicos (Duran et al., 2015). Esta modalidad puede darse mediante una interacción en tiempo real, a la cual se le conoce como sincrónica (videoconferencias y mensajería instantánea) (Castañeda y Vargas, 2021) y de manera asincrónica o diferida, la cual se caracteriza porque la interacción no se da en tiempo real (foros, wikis, tareas, etc.) (Herrera et al., 2019). Sin embargo, la pandemia provocó que a partir de la virtualización obligatoria en la educación 
surjan algunos problemas, tanto en los docentes como en los estudiantes, entre los que destaca el tecnoestrés.

El concepto de tecnoestrés fue señalado por primera vez por Brod (1982) hace casi 4 décadas y fue definido como una condición resultante de una incapacidad individual para adaptarse de manera saludable al uso de las nuevas tecnologías, que se modula según la edad, el tiempo de conexión, las experiencias tecnológicas previas, la carga de tareas y la percepción de control. Ello puede afectar el desempeño de las personas, limitando así el utilización (Estrada-Muñoz et al., 2020). Una década después, también se conceptualizó al tecnoestrés como el impacto negativo en actitudes, pensamientos, comportamientos o fisiología causado, directa o indirectamente, por usar tecnologías (Weil y Rosen, 1997). Posteriormente, Tarafdar et al. (2007) brindaron una las definiciones más usadas y extendidas que precisaba que el tecnoestrés se trataba del estrés creado por el uso de las TIC y que era producido por los intentos que tienen las personas por lidiar con las mencionadas tecnologías así como los cambios en las necesidades cognitivas y sociales relacionadas a su uso.

De manera general, tecnoestrés puede tener diferentes consecuencias en las personas que lo padecen. Estas pueden ser fisiológicas, tales como la somnolencia, falta de concentración, dolores oculares y de cabeza, dolencias musculares y problemas gastrointestinales (Salanova et al., 2007). En cuanto a las consecuencias psicosociales, el tecnoestrés puede ocasionar ansiedad, depresión, estrés y puede llegar a provocar a largo plazo burnout en el caso que la persona que lo padece no reciba tratamiento (Ragu et al., 2008). En el ámbito académico puede provocar la disminución del rendimiento académico de los estudiantes y en casos extremos, la deserción universitaria (Upadhyaya y Vrinda, 2021).

Según Dias y Costa (2008) y el Observatorio Permanente de Riesgos Psicosociales (2008) existen 3 tipos de tecnoestrés: Tecnoansiedad, tecnoadicción y tecnofatiga. La tecnoansiedad se refiere al estado en el que las personas sienten elevados niveles de activación fisiológica, aumento de la tensión y malestar por la utilización actual o futura de las TIC. La tecnoadicción es un fenómeno que se caracteriza por la constante necesidad de utilizar de manera obsesiva y compulsiva las TIC, en todo lugar y momento. Por último, la tecnofatiga se caracteriza por el cansancio y agotamiento cognitivo como consecuencia de la utilización de las TIC y puede agudizarse por la presencia de sensaciones de desconfianza e ineficacia 
frente a su uso. En virtud a lo expuesto, los rasgos del tecnoestrés pueden evidenciarse, tanto en los tecnófobos (personas que se resisten al uso de las TIC) y los tecnoadictos (personas que son dependientes a las TIC) (Picón et al., 2016).

Actualmente, la realidad en la región Madre de Dios, lugar donde se realizó la investigación, es bastante compleja. Para educarse virtualmente, los estudiantes tienen que participar de las sesiones de aprendizaje mediante videoconferencias, utilizando principalmente Google Meet, Zoom, WebEx y Microsoft Tems durante largos periodos. Además deben cumplir con sus responsabilidades académicas encomendadas por sus docentes (informes, monografías, ensayos, lecturas, resúmenes, etc.) lo cual provoca una sobreexposición a las TIC, es decir, que se encuentren hiperconectados. Sumado a ello, tienen que lidiar con los problemas de conectividad y acceso a las TIC, situación que podría incrementar la prevalencia del tecnoestrés, repercutiendo, tal como se mencionó, en su salud, bienestar emocional y desempeño académico.

Aunque el tecnoestrés ya ha sido estudiado en otros contextos y a pesar de las consecuencias negativas asociadas a su prevalencia, existen pocas investigaciones sobre este tema en el campo de la educación, particularmente en la educación superior durante la pandemia. Así pues, Upadhyaya y Vrinda (2021) realizaron un estudio en una universidad privada de la India y determinaron que los estudiantes experimentaron niveles moderados de tecnoestrés durante el desarrollo de las clases virtuales, lo cual tuvo un impacto negativo en su productividad académica. Del mismo modo, Montes de Oca et al. (2021) desarrollaron una investigación en estudiantes universitarios mexicanos para medir el grado de tecnoestrés y concluyeron que se presentaba en un nivel moderado, representando un riesgo psicológico latente que debía ser tomado en cuenta puesto que tenía efectos en la salud física, emocional y psicológica en las personas que lo padecían. Por otro lado, Oladosu et al. (2021) realizaron un estudio en una universidad pública de Nigeria para conocer la prevalencia del tecnoestrés y conocer su influencia en el aprendizaje de estudiantes. Concluyeron que a medida que los estudiantes universitarios usaban dispositivos inteligentes, se volvían tecnológicamente estresados y esto repercutía negativamente en su aprendizaje. Finalmente Wang et al. (2020) realizaron un estudio en dos universidades públicas de China con el propósito de describir el tecnoestrés entre los estudiantes universitarios en el aprendizaje 
mejorado por la tecnología. Hallaron que existía una prevalencia regular de tecnoestrés que provocaba agotamiento y a su vez afectaba negativamente su desempeño percibido.

Teniendo en cuenta lo manifestado, el objetivo de la presente investigación fue describir el tecnoestrés de los estudiantes de la carrera profesional de Educación de una universidad pública de la Amazonía peruana durante la pandemia COVID-19.

\section{Desarrollo \\ Metodología}

La investigación tuvo un enfoque cuantitativo, ya que para contrastar las hipótesis se realizó la recolección de datos, lo que permitió medir numéricamente la información que se obtuvo para realizar su sistematización y análisis (Sánchez et al., 2018); el diseño fue no experimental, puesto que la variable tecnoestrés no fue manipulada intencionalmente, sino solo fue observada (Hernández et al., 2014) y el tipo de investigación fue descriptivo transeccional, ya que se analizaron las características de la variable tecnoestrés y la recolección de datos fue realizada en un solo momento (Bisquerra, 2009).

\section{Población y muestra}

La investigación se realizó en la Facultad de Educación de la Universidad Nacional Amazónica de Madre de Dios de la ciudad de Puerto Maldonado, Perú. La población estuvo constituida por 586 estudiantes matriculados en el ciclo 2021 I mientras que la muestra fue conformada por 232 estudiantes, cantidad obtenida mediante un muestreo probabilístico estratificado con un nivel de confianza del 95\% y un nivel de significancia del 5\%. En la tabla 1 se describen las características sociodemográficas y académicas de la muestra. Así pues, el 46,6\% de los estudiantes eran de la especialidad de Inicial y Especial, el 35,3\% de Primaria e Informática y el 18,1\% de Matemática y Computación. Respecto al sexo, el 56,5\% de los participantes eran mujeres y el $43,5 \%$ eran varones. En cuanto al grupo etario, el 49,6\% tenía entre 16 y 20 años, el 31,5\% entre 22 y 25 años, el 13,8\% entre 26 y 30 años y el 5,1\% de los estudiantes tenían más de 30 años de edad. Por otro lado, el rendimiento académico del 50,9\% de participantes era bueno, del 44,8\% era regular y del 4,3\% era malo. Finalmente, con relación a la condición laboral, el $58,2 \%$ de los estudiantes trabajaba y el $41,8 \%$ no trabajaba. 
Tabla 1 - Características de la muestra.

\begin{tabular}{|c|c|c|c|}
\hline \multicolumn{2}{|c|}{ Variables sociodemográficas y académicas } & $\mathrm{n}=232$ & $\%$ \\
\hline \multirow{3}{*}{ Especialidad } & Inicial y Especial & 108 & 46,6 \\
\hline & Primaria e Informática & 82 & 35,3 \\
\hline & Matemática y Computación & 42 & 18,1 \\
\hline \multirow{2}{*}{ Sexo } & Varón & 101 & 43,5 \\
\hline & Mujer & 131 & 56,5 \\
\hline \multirow{4}{*}{ Grupo etario } & Entre 16 y 20 años & 115 & 49,6 \\
\hline & Entre 21 y 25 años & 73 & 31,5 \\
\hline & Entre 26 y 30 años & 32 & 13,8 \\
\hline & Más de 30 años & 12 & 5,1 \\
\hline \multirow{3}{*}{$\begin{array}{l}\text { Rendimiento } \\
\text { académico }\end{array}$} & Bueno & 118 & 50,9 \\
\hline & Regular & 104 & 44,8 \\
\hline & Malo & 10 & 4,3 \\
\hline Condición & Trabaja & 97 & 41,8 \\
\hline laboral & No trabaja & 135 & 58,2 \\
\hline
\end{tabular}

Fuente: Dirección Universitaria de Asuntos Académicos.

\section{Técnicas e instrumentos}

Para realizar la recolección de datos se recurrió a la técnica de la encuesta mientras que el instrumento utilizado fue el Cuestionario de Tecnoestrés, el cual fue construido por Cazares (2019), sin embargo, debido a que la versión original estaba dirigida a la población laboral, fue necesario adaptarla a la población universitaria. Está conformado por 20 reactivos de tipo Likert (nunca, casi nunca, a veces, casi siempre y siempre) los cuales evalúan 3 dimensiones: tecnoansiedad (ítems del 1 al 9), tecnoadicción (ítems del 10 al 17) y tecnofatiga (ítems del 18 al 20). Sus propiedades métricas se determinaron mediante los procesos de validez y confiabilidad. La validación de contenido se realizó a través de la técnica de juicio de expertos, donde luego de su evaluación se obtuvo un coeficiente $\mathrm{V}$ de Aiken de 0,924; lo que significaba que el cuestionario tenía un muy adecuado nivel de validez. En cuanto a la confiabilidad, fue determinada a través de una prueba piloto realizada a 25 estudiantes, obteniéndose un coeficiente Alfa de Cronbach de 0,942; lo que indicaba que la consistencia interna del instrumento también era muy adecuada.

\section{Procedimiento}

La recolección de datos se dio en el mes de junio del año 2021. Para ello se solicitó la autorización a la institución 
universitaria. Una vez obtenida la misma, se contactó a los estudiantes mediante la aplicación de mensajería instantánea WhatsApp ${ }^{\circledR}$ con la finalidad de explicarles el propósito de la investigación y enviarles en enlace para que puedan acceder al formulario creado en la página de Google ${ }^{\circledR}$. Posteriormente, los estudiantes accedieron al formulario, leyeron las orientaciones, brindaron su consentimiento y procedieron a responder el cuestionario cuya duración fue de 12 minutos aproximadamente.

\section{Análisis de datos}

El acceso al formulario se cerró al recepcionar las 232 respuestas y para realizar el análisis estadístico se recurrió al
Software SPSS®, donde los resultados descriptivos se sistematizaron en tablas de frecuencia y porcentaje. En cuanto a la parte inferencial, fue realizada utilizando la prueba no paramétrica Chi Cuadrado $\left(\mathrm{X}^{2}\right)$ con el propósito de determinar si el tecnoestrés se asociaba de manera significativa con las variables sociodemográficas y académicas propuestas.

\section{Resultados}

Posterior a la aplicación del cuestionario se estructuró una base de datos, donde las variables y dimensiones fueron categorizadas y se elaboraron las tablas que se muestran a continuación.

Tabla 2 - Resultado descriptivo de la variable tecnoestrés y sus dimensiones.

\begin{tabular}{lcccccccc}
\hline \multirow{2}{*}{ Niveles } & \multicolumn{2}{c}{ Tecnoestrés } & \multicolumn{2}{c}{ Tecnoansiedad } & \multicolumn{2}{c}{ Tecnoadicción } & \multicolumn{2}{c}{ Tecnofatiga } \\
\cline { 2 - 9 } & $\mathrm{n}$ & $\%$ & $\mathrm{n}$ & $\%$ & $\mathrm{n}$ & $\%$ & $\mathrm{n}$ & $\%$ \\
\hline Alto & 50 & 21,6 & 47 & 20,3 & 41 & 17,7 & 58 & 25,0 \\
Moderado & 98 & 42,2 & 79 & 34,0 & 126 & 54,3 & 94 & 40,5 \\
Bajo & 84 & 36,2 & 106 & 45,7 & 65 & 28,0 & 80 & 34,5 \\
Total & 232 & 100,0 & 232 & 100,0 & 232 & 100,0 & 232 & 100,0 \\
\hline
\end{tabular}

Fuente: Base de datos.

De acuerdo a la tabla 2, el 42,2\% de los estudiantes presentó un nivel moderado de tecnoestrés, el 36,2\% tenía un nivel bajo y el 21,6\% evidenciaba un nivel alto. Este hallazgo indica la existencia del tecnoestrés en diferentes niveles en los estudiantes, lo cual ya es una señal de preocupación puesto que muchos de ellos están sobreexpuestos a las computadoras, laptops, tablets y celulares para participar de las clases y realizar sus trabajos. Ello podría traer secuelas en los diferentes aspectos de su vida (personal, familiar, académico, social), tales como 
somnolencia, falta de concentración, los dolores oculares y de cabeza, dolencias musculares, problemas gastrointestinales, ansiedad, depresión, estrés y hasta burnout académico en el caso que no reciban un tratamiento oportuno.

Respecto a la primera dimensión, se halló que el 45,7\% de los estudiantes presentaba un bajo nivel de tecnoansiedad, el 34\% tenía un nivel moderado y el 20,3\% evidenciaba un nivel alto (tabla 2). Ello indica que los estudiantes se caracterizan porque, a pesar de estar hiperconectados, no habían desarrollado fobias, pensamientos negativos 0 una indisposición para utilizar ahora o en el futuro algún tipo de TIC.

En cuanto a la segunda dimensión, se determinó que el 54,3\% de los estudiantes tenía un nivel moderado de tecnoadicción, el $28 \%$ presentaba un nivel bajo y el $17,7 \%$ mostraba un nivel alto (tabla 2). La información expuesta indica que en muchos casos los estudiantes han desarrollado una compulsión por utilizar las TIC durante periodos prolongados $\mathrm{y}$ han desarrollado cierta dependencia hacia su uso.

Finalmente, con relación a la tercera dimensión, se encontró que el 40,5\% había desarrollado un nivel moderado de tecnofatiga, el 34,5\% evidenciaba un nivel bajo y el 25\% presentaba un nivel alto (tabla 2). En ese sentido, los estudiantes se caracterizaban porque tenían sentimientos de cansancio y agotamiento emocional y cognitivo ocasionado por el uso prolongado o desmedido de las TIC, lo cual impactaría también en sus pensamientos y actitudes.

Tabla 3 - Tecnoestrés y variables sociodemográficas y académicas.

\begin{tabular}{|c|c|c|c|c|c|c|}
\hline \multirow{2}{*}{\multicolumn{2}{|c|}{ Variables sociodemográficas y académicas }} & \multicolumn{3}{|c|}{ Tecnoestrés } & \multirow{2}{*}{$X^{2}$} & \multirow{2}{*}{ p-valor } \\
\hline & & Alto & Moderado & Bajo & & \\
\hline \multirow{3}{*}{ Especialidad } & Inicial y Especial & $16(14,8 \%)$ & $51(47,2 \%)$ & $41(38,0 \%)$ & \multirow{3}{*}{17,253} & \multirow{3}{*}{0,066} \\
\hline & Primaria e Informática & $21(25,6 \%)$ & $32(39,0 \%)$ & $29(35,4 \%)$ & & \\
\hline & Matemática y Computación & $13(31,0 \%)$ & $15(35,7 \%)$ & $14(33,3 \%)$ & & \\
\hline \multirow{2}{*}{ Sexo } & Varón & $21(20,8 \%)$ & $40(39,6 \%)$ & $40(39,6 \%)$ & \multirow{2}{*}{20,031} & \multirow{2}{*}{0,003} \\
\hline & Mujer & $29(22,1 \%)$ & $58(44,3 \%)$ & $44(33,6 \%)$ & & \\
\hline \multirow{4}{*}{ Grupo etario } & Entre 16 y 20 años & $29(25,2 \%)$ & $48(41,7 \%)$ & $38(33,1 \%)$ & \multirow{4}{*}{27,935} & \multirow{4}{*}{0,042} \\
\hline & Entre 21 y 25 años & $12(16,5 \%)$ & $32(43,8 \%)$ & $29(39,7 \%)$ & & \\
\hline & Entre 25 y 30 años & 7 (21,9\%) & $13(40,6 \%)$ & $12(37,5 \%)$ & & \\
\hline & Más de 30 años & $2(16,6 \%)$ & $5(41,7 \%)$ & $5(41,7 \%)$ & & \\
\hline
\end{tabular}




\begin{tabular}{|c|c|c|c|c|c|c|}
\hline \multirow{3}{*}{$\begin{array}{l}\text { Rendimiento } \\
\text { académico }\end{array}$} & Bueno & $24(20,3 \%)$ & $53(44,9 \%)$ & $41(34,8 \%)$ & \multirow{3}{*}{10,354} & \multirow{3}{*}{0,052} \\
\hline & Regular & $22(21,2 \%)$ & 43 (41,3\%) & $39(37,5 \%)$ & & \\
\hline & Malo & $4(40,0 \%)$ & $2(20,0 \%)$ & $4(40,0 \%)$ & & \\
\hline Condición & Trabaja & $28(28,9 \%)$ & $41(42,2 \%)$ & $28(28,9 \%)$ & \multirow{2}{*}{7,783} & \multirow{2}{*}{0,011} \\
\hline laboral & No trabaja & $22(16,3 \%)$ & 57 (42,2\%) & $56(41,5 \%)$ & & \\
\hline
\end{tabular}

Fuente: Base de datos.

La información expuesta en la tabla 3 indica que el sexo, el grupo etario y la condición laboral de los estudiantes se asociaron de manera significativa con los niveles de tecnoestrés $(\mathrm{p}<0,05)$, no obstante, no se hallaron asociaciones significativas con la especialidad y el rendimiento académico $(\mathrm{p}>0,05)$. En ese sentido, se pudo establecer que las mujeres que eran más jóvenes y trabajaban presentaron niveles ligeramente superiores de tecnoestrés en comparación con los otros grupos de contraste.

\section{Discusión}

En la actualidad, las distintas sociedades vienen afrontando la pandemia de COVID-19, la cual ha provocado grandes cambios y repercusiones en la educación, motivando a una migración obligatoria de la presencialidad a la virtualidad. A pesar que se sabe que la educación virtual tiene muchas ventajas como una mayor cobertura, flexibilidad en los horarios y la integración de las TIC en el proceso de enseñanza, la coyuntura hizo que su implementación sea de manera poco adecuada, trayendo consigo problemas como el tecnoestrés. En ese orden de ideas, la presente investigación describió el tecnoestrés percibido por los estudiantes universitarios peruanos y determinó también algunas variables sociodemográficas que estaban asociadas a su prevalencia.

Un primer hallazgo indica que el nivel predominante de tecnoestrés en los estudiantes universitarios era moderado. Estos hallazgos muestran que la existencia de una prevalencia que debe ser tomada en cuenta dadas las implicancias que tiene este fenómeno, no solo en el aspecto académico, sino personal, familiar y social. Ahora bien, el nivel de tecnoestrés sería ocasionado principalmente por la continua exposición de los estudiantes a las TIC (computadoras, laptops, tablets $\mathrm{y}$ celulares) para participar de las actividades sincrónicas y asincrónicas, que provocaría altos niveles cansancio y agotamiento mental. Lo expuesto es corroborado con un hallazgo emergente también encontrado en el presente estudio que indica que los estudiantes se caracterizaban por tener 
niveles moderados de tecnofatiga $y$ tecnoadicción. Finalmente, se puede agregar que la conectividad, caracterizada por su intermitencia, también pudo haber contribuido a agudizar la problemática mencionada.

El hallazgo expuesto coincide con lo reportado por Upadhyaya y Vrinda (2021) quienes realizaron un estudio en una universidad privada de la India y determinaron que los estudiantes experimentaron niveles moderados de tecnoestrés durante el desarrollo de las clases virtuales en el contexto de la pandemia, lo cual tuvo un impacto negativo en su productividad académica. Asimismo, es coherente con los resultados de Montes de Oca et al. (2021), que desarrollaron una investigación en estudiantes universitarios mexicanos para medir el grado de tecnoestrés y concluyeron que se presentaba en un nivel moderado, representando un riesgo psicológico latente que debía ser tomado en cuenta puesto que tenía efectos en la salud física, emocional y psicológica en las personas que lo padecían. Del mismo modo, guarda relación con el estudio de Oladosu et al. (2021), quienes realizaron un estudio en una universidad pública de Nigeria para conocer la prevalencia del tecnoestrés y determinar su influencia en el aprendizaje de estudiante, llegando a concluir que a medida que los estudiantes universitarios usaban con mayor frecuencia los dispositivos inteligentes, se volvían tecnológicamente más estresados y esto repercutía desfavorablemente en su aprendizaje. Finalmente, es similar a los resultados de Wang et al. (2020), que realizaron un estudio en dos universidades públicas de China con el propósito de describir el tecnoestrés entre los estudiantes universitarios en el aprendizaje mejorado por la tecnología. Hallaron que existía una prevalencia regular de tecnoestrés, la cual provocaba agotamiento y a su vez afectaba negativamente su desempeño percibido.

El uso cada vez más extendido y frecuente de las TIC ha provocado la aparición de fenómenos nocivos para los usuarios como el tecnoestrés, que es una enfermedad adaptativa provocada por la falta de capacidad para afrontar dichas tecnologías de manera saludable (Penado et al., 2020). Entonces, la convivencia cada vez más frecuente de las personas con la tecnología puede provocar un impacto negativo en las actitudes, pensamientos $\mathrm{y}$ comportamientos que ellas tienen. En la actualidad, el tecnoestrés viene siendo estudiado para conocer sus causas y efectos negativos (Özgür, 2020), está presentándose con mayor frecuencia durante la pandemia debido al uso 
frecuente de las TIC y sus síntomas son la ansiedad, enfermedades físicas, tensión conductual, tecnofobia, fatiga mental, alteraciones de la memoria, falta de concentración, irritabilidad y sensaciones de agotamiento e insomnio (Molino, et al., 2020).

En cuanto a las variables sociodemográficas, se determinó que la prevalencia del tecnoestrés estaba asociada de manera significativa con el sexo de los estudiantes $(\mathrm{p}<0,05)$. En ese sentido, se observa que las mujeres presentaron niveles ligeramente superiores de tecnoestrés que los varones, lo cual podría ser ocasionado porque ellas suelen padecer de trastornos interiorizantes como la depresión y la ansiedad frente a situaciones estresantes y exteriorizan más las manifestaciones emocionales y fisiológicas producidas por el estrés (Estrada et al., 2020a). Asimismo, muchas de ellas cumplen labores domésticas además de laborales, lo cual incrementa sus responsabilidades.

El hallazgo descrito coincide con múltiples investigaciones que determinaron que las mujeres presentaron mayores niveles de tecnoestrés en comparación a los varones (Salanova et al., 2007; Dias y Costa, 2008; Carlotto, 2010; Carlotto y Gonçalves, 2010; Çoklar y Şahin, 2011; Picón et al., 2016; Villavicenco et al.,
2020; Wang et al., 2020), sin embargo, estos resultados no son concluyentes, ya que algunas investigaciones determinaron que eran los hombres quienes estaban más propensos a padecer dicha enfermedad (Ragu et al., 2008; Coppari et al., 2017).

Por otro lado, se determinó que los niveles de tecnoestrés que presentaban los estudiantes estaban asociados significativamente con el grupo etario al que pertenecían $(\mathrm{p}<0,05)$. Bajo esa premisa, se encontró que los estudiantes más jóvenes tenían mayores niveles de tecnoestrés que los estudiantes pertenecientes a los grupos etarios de mayor edad. Este hallazgo discrepa de lo pensado inicialmente $\mathrm{y}$ es similar al resultado de una investigaciones realizada en Argentina (Picón et al., 2016). No obstante, difiere de otros estudios donde concluyeron que a medida que pasan los años, se incrementan la prevalencia del tecnoestrés, es decir, se incrementan los niveles de tecnofatiga, tecnoansiedad, escepticismo y se presentan creencias más consistentes de ineficacia en el uso de las TIC. (Salanova et al., 2007; Dias y Costa, 2008; Carlotto et al., 2017; Özgür, 2020; Sánchez et al., 2020).

Asimismo, se determinó que la prevalencia del tecnoestrés se asociaba de manera significativa con la condición laboral de los estudiantes $(\mathrm{p}<0,05)$. En ese 
entender, las personas que además de cumplir su rol de estudiante trabajaban, presentaron niveles ligeramente superiores de tecnoestrés en comparación a las personas que solo se dedicaban a estudiar. Ello es lógico, ya que se demostró que existía una prevalencia considerable de tecnofatiga, la cual originaba que los estudiantes lleguen cansados a las clases virtuales debido al cumplimiento de sus responsabilidades laborales y sumado a las responsabilidades académicas asignadas por sus docentes, generaron en ellos un desajuste emocional.

El resultado expuesto es coherente con los hallazgos encontrados por Estrada et al. (2020b) quienes determinaron que los niveles del burnout académico (respuesta fisiológica a niveles crónicos de estrés) estaban asociados de manera significativa a la situación laboral de los estudiantes, lo cual era explicado porque ellos se preocupaban más por gestionar su tiempo y cumplir con sus responsabilidades de manera oportuna que a su vez generaba niveles elevados de estrés.

De acuerdo a Alcas et al. (2019), en los últimos años las instituciones educativas universitarias han ido incorporando las TIC para fortalecer sus procesos de enseñanza - aprendizaje, sin embargo, este proceso no conduce de manera inmediata a la optimización de la práctica educativa. Supone un proceso de implementación complejo e implica una capacidad de respuesta por parte de los involucrados (principalmente docentes y estudiantes) debido a la alta y continua exposición a dichas tecnologías. Entonces, los estudiantes, en el caso de que no puedan cumplir cabalmente con las exigencias académicas, podrían desarrollar sentimientos de angustia, ansiedad, fatiga y posteriormente desencadenaría en un desajuste psicológico y fisiológico. Ello afectaría su calidad de vida, bienestar psicológico e influiría en su rendimiento académico. Por ello, resulta imperativo la detección del tecnoestrés y una oportuna intervención.

A pesar que en la presente investigación se realizaron hallazgos importantes, es menester precisar algunas limitaciones. En primer lugar, la cantidad y homogeneidad de estudiantes, lo cual no permite generalizar los resultados. En segundo lugar, el instrumento de recolección de datos fue autoadministrado, lo cual podría haber generado sesgos de deseabilidad social. Por ello, se sugiere que los futuros estudios incrementen la cantidad de participantes y procuren su heterogeneidad para que los hallazgos puedan generalizarse. Del mismo modo, sería importante complementar la 
recolección de datos mediante la aplicación de otras técnicas como la entrevista.

\section{Consideraciones finales}

Durante la emergencia sanitaria, el proceso de enseñanza en la educación superior cambió de modalidad, es decir, se dio de manera virtual mediante la utilización de las TIC. Sin embargo, esta forma de enseñar, a pesar de sus múltiples beneficios, trajo consigo algunas consecuencias negativas para los estudiantes como el tecnoestrés. En ese sentido, en la presente investigación se halló que los estudiantes se caracterizaban por tener niveles moderados de tecnoestrés. Respecto a sus dimensiones, se encontró que existían niveles bajos de tecnoansiendad y niveles moderados de tecnoadicción y tecnofatiga. Finalmente, se determinó que algunas variables sociodemográficas como el sexo, el grupo etario y la condición laboral de los estudiantes se asociaba significativamente a los niveles de tecnoestrés. Como se puede ver, los hallazgos expuestos son muy relevantes porque permitieron comprender cómo afecta el tecnoestrés a los estudiantes en el contexto de la pandemia COVID-19 y qué variables se asocian a su prevalencia. En virtud a lo expuesto, es necesario que la universidad tome algunas medidas preventivas $\mathrm{y}$ correctivas que permitan disminuir la prevalencia de dicho padecimiento los estudiantes. Asimismo, sería importante fomentar la desconexión digital para que ellos realicen actividades físicas, de atención a sus familias y socialización.

\section{Referencias}

Alcas, N., Alarcón, H., Venturo, C., Alarcón, M., Fuentes, J., \& López, T. (2019). Tecnoestrés docente y percepción de la calidad de servicio en una universidad privada de Lima. Propósitos y Representaciones, 7(3), 231-247. http://dx.doi.org/10.20511/pyr2019.v7n3.3 $\underline{88}$

Bisquerra, R. (2009). Metodología de la Investigación Educativa (2a ed.). Madrid: La Muralla S.A.

Brod, C. (1984). Technostress: The human cost of the computer revolution. USA: Addison-Wesley Publishing Company.

Buitrago, F., Ciurana, R., Fernández, M., \& Tizón, J. (2021). Pandemia de la COVID-19 y salud mental: reflexiones iniciales desde la atención primaria de salud española. Atención Primaria, 53(1), 89-101.

https://doi.org/10.1016/j.aprim.2020.06.00 $\underline{6}$

Carlotto, S. (2010). Fatores de risco do tecnoestresse em trabalhadores que utilizam tecnologias de informação e comunicação. Estudos de Psicologia, 15(3), 319-324. https://doi.org/10.1590/S1413294X2010000300012

Carlotto, S., Wendt, G., \& Jones, A. (2017). Technostress, career commitment, satisfaction with life, and work-family 
interaction among workers in information and communication technologies. Actualidades en Psicología, 31(122), 91102.

https://doi.org/10.15517/ap.v31i122.22729

Carlotto, S., \& Gonçalves, S. (2010). O tecnoestresse em trabalhadores que atuam com tecnologia de informação e comunicação. Psicologia, Ciência $e$ Profissão, 30(2), 308-317. https://doi.org/10.1590/S141498932010000200007

Castañeda, K., \& Vargas, A. (2021). En tiempos de pandemia: una mirada retrospectiva sobre la educación a distancia, virtual y remota de emergencia, así como sobre las buenas prácticas docentes. Academia y Virtualidad, 14(1), 13-22. https://doi.org/10.18359/ravi.5346

Cazares, M. (2019). Adaptación de dos escalas para medir tecnoestrés y tecnoadicción en una población laboral mexicana (Tesis de Pregrado). Universidad Nacional Autónoma de México, México.

Coppari, N., Bagnoli, L., Codas, G., Montanía, M., Martínez, U., \& López, H. (2017). Uso de tecnologías de la comunicación e información y tecnoestrés en estudiantes paraguayos: su relación con la edad. Revista Cuadernos de Neuropsicología, 11(3), 166-181. https://www.cnps.cl/index.php/cnps/article/ view/306

Çoklar, A., \& Şahin, Y. (2011) Technostress levels of social network users based on ICTs in Turkey. European Journal of Social Sciences, 23(2), 171-182. https://www.tecnostress.it/wpcontent/uploads/2011/09/Technostress-inSocial-Turkey.pdf

Dias, M., \& Costa, J. (2008). Impacto psicosocial de la tecnología de información y comunicación (TIC): tecnoestrés, daños físicos y satisfacción laboral. Acta
Colombiana de Psicología, 11(2), 127-139. https://actacolombianapsicologia.ucatolica. edu.co/article/view/307

Díaz, P., Andrade, Hincapié, A., \& Uribe, A. (2021). Análisis del proceso metodológico en programas de educación superior en modalidad virtual. Revista de Educación a Distancia (RED), 21(65), 141. https://doi.org/10.6018/red.450711

Duran, R., Estay, C., \& Álvarez, H. (2015). Adopción de buenas prácticas en la educación virtual en la educación superior. Aula Abierta, 43(2), 77-86. https://doi.org/10.1016/j.aula.2015.01.001

Estrada, E., Gallegos, N., Mamani, H., \& Huaypar, K. (2020a). Actitud de los estudiantes universitarios frente a la educación virtual en tiempos de la pandemia de COVID-19. Revista Brasileira de Educação do Campo, 5, e10237.

https://doi.org/10.20873/uft.rbec.e10237

Estrada, E., Gallegos, N., \& Mamani, H. (2020b). Burnout académico en estudiantes universitarios peruanos. Apuntes Universitarios, 11(2), 48-62. https://doi.org/10.17162/au.v11i2.631

Estrada, E., \& Mamani, M. (2021). Competencia digital y variables sociodemográficas en docentes peruanos de educación básica regular. Revista San Gregorio, 1(45), 1-16. http://dx.doi.org/10.36097/rsan.v0i45.1502

Estrada-Muñoz, C., Castillo, D., VegaMuñoz, A., \& Boada-Grau, J. (2020). Teacher technostress in the Chilean school system. International Journal of Environmental Research and Public Health, 17(15), 1-17. https://doi.org/10.3390/ijerph17155280

Hernández, R., Fernández, C., \& Baptista, P. (2014). Metodología de la Investigación (6 ed.). México: Mc Graw-Hill. 
Herrera, C., Espinoza, M., Ludeña., V., \& Michay, G. (2019). Las Tics como herramienta de interacción y colaboración en el área de Biología. Revista Espacios, 40(41), $1-10$. https://www.revistaespacios.com/a19v40n 41/19404101.html

Lizcano, F., \& Arroyave, F. (2020). El ambiente, los desplazamientos y el riesgo cardiovascular en la pandemia por COVID-19. Revista Colombiana de Cardiología, 27(3), 160-165. https://doi.org/10.1016/j.rccar.2020.05.001

Mejía, C., Rodríguez, J., Charri, J., Liendo, D., Morocho, N., Benites, C., Avalos, M., Medina, D., Carranza, R., \& Mamani, O. (2021). Repercusión académica de la COVID-19 en universitarios peruanos. Revista Cubana de Investigaciones Biomédicas, $\quad 40(1), \quad 16$. http://www.revibiomedica.sld.cu/index.php /ibi/article/view/814

Mojica, R., \& Morales, M. (2020). Pandemia COVID-19, la nueva emergencia sanitaria de preocupación internacional: una revisión. Medicina de Familia. SEMERGEN, 46(1), 65-77. https://doi.org/10.1016/j.semerg.2020.05.0 $\underline{10}$

Molino, M., Ingusci, E., Signore, F., Manuti, A., Giancaspro, M., Russo, V., Zito, M., \& Cortese, C. (2020). Wellbeing costs of technology use during Covid-19 remote working: An investigation using the Italian Translation of the Technostress Creators Scale. Sustainability, 12(15), 5911. https://doi.org/10.3390/su12155911

Montes de Oca, J., Alcántara, S., \& Domínguez, A. (2021). Tecnoestrés en docentes y alumnos universitarios: medición en tiempos de COVID19. Revista de Desarrollo Sustentable, Negocios, Emprendimiento y Educación, 16, 98-109. https://www.eumed.net/es/revistas/rilcoDS /16-febrero21/tecnoestres-docentes-

$\underline{\text { alumnos }}$

Murillo, F., \& Duk, C. (2020). El Covid-19 y las brechas educativas. Revista Latinoamericana de Educación Inclusiva, 14(1), 11-13. http://dx.doi.org/10.4067/S0718$\underline{73782020000100011}$

Observatorio Permanente de Riesgos Psicosociales. (2008). Tecnoestrés. Efecto sobre la salud y prevención. Madrid: Comisión Ejecutiva Confederal de UGT.

Oladosu, K., Alasan, N., Ibironke, E., Ajani, H., \& Jimoh, T. (2021). Learning with Smart Devices: Influence of Technostress on Undergraduate Students' Learning at University of Ilorin, Nigeria. International Journal of Education and Development using Information and Communication Technology, 16(2), 40-47. http://ijedict.dec.uwi.edu/viewarticle.php?i $\underline{\mathrm{d}=2757}$

Ordorika, I. (2020). Pandemia y educación superior. Revista de la Educación Superior, $\quad 49(194)$, 1-8. https://doi.org/10.36857/resu.2020.194.112 $\underline{0}$

Özgür, H. (2020). Relationships between teachers' technostress, technological pedagogical content knowledge (TPACK), school support and demographic variables: A structural equation modeling. Computers in Human Behavior, 112, 106468. https://doi.org/10.1016/j.chb.2020.106468

Penado, M., Rodicio, M., Ríos, M., \& Mosquera, M. (2020). Technostress in Spanish University Students: Validation of a measurement scale. Frontiers of Psychology, 11, 1-9. https://dx.doi.org/10.3389\%2Ffpsyg.2020. $\underline{582317}$

Picón, C., Toledo, S., \& Navarro, V. (2016). Tecnoestrés: Identificación y 
prevalencia en el personal docente de la Facultad de Medicina de la Universidad Nacional del Nordeste. Revista de la Facultad de Medicina de la UNNE, 36(3), 41-51.

http://revista.med.unne.edu.ar/index.php/m ed/article/view/14

Quiroz, C., Pareja, A., Valencia, E., Enríquez, Y., De Leon, J., \& Aguilar, P. (2020). Un nuevo coronavirus, una nueva enfermedad: COVID-19. Horizonte Médico (Lima), 20(2), e1208. https://doi.org/10.24265/horizmed.2020.v2 0n2.11

Ragu, T., Tarafdar, M., Ragu, B., \& Tu, Q. (2008). The consequences of technostress for end users in organizations: Conceptual development and empirical validation. Information Systems Research, 19(4), 417433. https://doi.org/10.1287/isre.1070.0165

Salanova, M., Llorens, S., Cifre, E., \& Nogareda, C. (2007). Tecnoestrés: Concepto, medida e intervención psicosocial. España: Instituto Nacional de Seguridad e Higiene en el Trabajo.

Sánchez, H., Reyes, C., \& Mejía, K. (2018). Manual de términos en investigación científica, tecnológica y humanística. Lima: Universidad Ricardo Palma.

http://repositorio.urp.edu.pe/handle/URP/1 $\underline{480}$

Sánchez, M., Cebrián, B., Ferré, P., Navarro, M., \& Plazuelo, N. (2020). Tecnoestrés y edad: un estudio transversal en trabajadores públicos. Revista Cuadernos de Neuropsicología, 14(2), 2533. https://doi.org/10.7714/CNPS/14.2.203

Suárez, J., Bedoya, L., Posada, M., Arboleda, E., Urbina, A., Ramírez, S., Bohórquez, C., \& Ferreira, J. (2021). Percepción de los estudiantes sobre adaptaciones virtuales en cursos de anatomía humana por la contingencia
SARS-CoV-2. Academia y Virtualidad, 14(1), 151-168. https://doi.org/10.18359/ravi.5275

Tarafdar, M., Tu, Q., Ragu, B., \& Ragu, T. (2007). The impact of technostress on role stress and productivity. Journal of Management Information Systems, 24(1), 301-328. https://doi.org/10.2753/MIS0742$\underline{1222240109}$

Toquero, C. (2020). Challenges and Opportunities for Higher Education amid the COVID-19 Pandemic: The Philippine Context. Pedagogical Research, 5(4), em0063. https://doi.org/10.29333/pr/7947

Upadhyaya, P., \& Vrinda (2021). Impact of technostress on academic productivity of university students. Education and Information Technologies, 26, 1647-1664. https://doi.org/10.1007/s10639-020-10319$\underline{9}$

Villavicencio, E., Ibarra, D., \& Calleja, N. (2020). Tecnoestrés en población mexicana y su relación con variables sociodemográficas y laborales. Psicogente, 23(44), 1-27. https://doi.org/10.17081/psico.23.44.3473

Wang, X., Tan, C., \& Li, L. (2020). Technostress in university students' technology-enhanced learning: An investigation from multidimensional person-environment misfit. Computers in Human Behavior, 105, 106208. https://doi.org/10.1016/j.chb.2019.106208

Weil, M., \& Rosen, L. (1997). Technostress: Coping with technology @WORK@HOME@PLAY. New York: Wiley.

Información del Artículo

Recibido en: 28/07/2021

Aprobado en: $23 / 08 / 2021$

Publicado en: $31 / 08 / 2021$ 
Contribuciones en el artículo: Edwin Gustavo Estrada Araoz: Fue responsable de la concepción, el diseño de la investigación, la recolección de datos, el análisis e interpretación de datos y la revisión final del artículo. Néstor Antonio Gallegos Ramos: Realizó la metodología, recolección de datos, su análisis e interpretación y revisó la última versión del manuscrito. Karl Herbert Huaypar Loayza: Realizó la introducción, analizó los datos y realizó la revisión final. Yolanda Paredes Valverde: Analizó los resultados, elaboró las conclusiones, adecuó al formato de la revista y dio la revisión final del artículo. Rosel Quispe Herrera: Desarrolló la discusión, formuló las conclusiones y revisó la versión final, e aprobación de la versión final publicada.

Conflictos de Intereses: Los autores han declarado que no existe conflicto de intereses con respecto a este artículo.

\section{Evaluación del artículo}

Revisión por pares

Agencia de Desarrollo

No tenía financiación.

\section{Cómo citar este artículo}

APA

Estrada-Araoz, E. G., Gallegos-Ramos, N. A., HuayparLoayza, K. H., Paredes-Valverde, Y., \& Quispe-Herrera, R. (2021). Tecnoestrés en estudiantes de una universidad pública de la amazonía peruana durante la pandemia COVID-19. Rev. Bras. Educ. Camp., 6, e12777. http://dx.doi.org/10.20873/uft.rbec.e12777

ABNT

ESTRADA-ARAOZ, E. G.; GALLEGOS-RAMOS, N. A.; HUAYPAR-LOAYZA，K. H.; PAREDES-VALVERDE, Y.; QUISPE-HERRERA, R. Tecnoestrés en estudiantes de una universidad pública de la amazonía peruana durante la pandemia COVID-19. Rev. Bras. Educ. Camp., Tocantinópolis, $\quad$ v. 6, e12777, 2021. http://dx.doi.org/10.20873/uft.rbec.e12777 